\title{
Practical Assessment on Water Crisis Based on PSR and DCE
}

\author{
JiaYi Li
}

\author{
North China Electric Power University, Baoding 071000, China; \\ 1274652182@qq.com
}

Keywords: PSR; DCE; Water Crisis

\begin{abstract}
In this paper, a model is established to measure the water scarcity stage and the future water source situation. Firstly, a practical measure model is set up to evaluate the risk of water shortage in a certain area. The potential influencing factors are turned into 14 indexes. A serial of equations are used to standardize the indexes. Meanwhile, the weight of each index is calculated based on Entropy Weight Method. In the construction stage of the model , PSR is adopted to ensure the rational classification of all chosen indexes ,DCE is used to make sure the dynamic nature of indexes are properly considered as well.
\end{abstract}

\section{Introduction}

Water is essential for life. One quarter of the world's population experience water scarcity due to the limited amount of fresh water. Physical scarcity and economic scarcity are considered as two primary causes of water scarcity. Stepping in the modern society, requirement of water resources for residential and agricultural are rapidly increasing as a result of the growing global population. At the same time, industrial consumption and pollution also bring a heavy burden on the supply of fresh water.

\section{Previous Researches}

There are some previous studies on calculating the bearing capacity of water. AHP [1] is utilized to simulate the effect caused by all possible factors, which include social, economy, population, resources, environmen, as well as cultural and history. For the estimation of the impact from different factors, Xiangjuan Zhang et al. introduced a hierarchical model based on the selection of large factors (or the standards).

However, all the weight of each index was calculated using AHP by experts scoring, which is considered less objective, and the existing experts scoring cannot be used by any other country or region.

As for the dynamic analysis of some factors such as population,GDP and technology, Wei Wang et al. researched analysis on water shortage degree and change trend based on integrated model of PSR and $\mathrm{DCE}_{\text {[2]. }}$.

All these studies contribute significantly to the question we are analyzing here. However, these studies are limited in that, some of them put forward a subjective way to estimate the impacts, and some of them introduced a dynamic way with too many indicators which focus on a certain area , while none of them have investigated a suitable model with high practical value.

\section{A Practical Model for Measurement of Water Providing Ability}

\subsection{Data Standardization}

Nondimensionalization is needed in the calculating process due to different dimensions of indexes and their large quantities. Assuming there are $k$ evaluation objects and $n$ evaluation indexes, top $m$ of them have a positive influence on the risk index, while the remaining $(n-m)$ of them have a negative influence. If we use $i$ to represent the evaluation objects, use $j$ to represent evaluation indexes, marking the $i-$ th evaluation indexes of the $j$ - th evaluation objects as $x_{i j}$, we can obtain $s\left(x_{i j}\right)$ of positive indexes by using (1).

$$
s(x i j)=\frac{x_{i j}-\min x}{\max x_{j}-\min x_{j}}, i=1,2, \ldots, k ; j=1,2, \ldots, m
$$

we can also obtain $s(x i j)$ of negative indexes in a similar way : 


$$
s(x i j)=\frac{1-x i j-\min x j}{\max x j-\min x j}, i=1,2, \ldots, k ; j=m+1, m+2, \ldots, n
$$

In equation (1)and (2),s(xij) refers to the standardized $x i j, s(x i j) \in[0,1]$. The smaller $s(x i j)$ is the less contribution it has to the risk, on the contrary, the bigger s(xij) is the more it contributes to the risk.

\subsection{Determination of Index Weight}

The weight of each index reflects the contribution of the index to the total target. In order to obtain an objective evaluation on all indexes , we decided to use Entropy Weight Method[5].

According to the definition and principle of entropy, the system may be in several different states, and the probability of the occurrence of each state can be referred as

$X_{j}(j=1,2,3, \ldots, m)$, then we can obtained the following equation:

$$
S^{\prime}=\frac{X j}{\sum_{j=1}^{n} X j} ; S j=\frac{-\sum_{j=1}^{n} S^{\prime} \ln \left(S^{\prime}\right)}{\ln (n)}
$$

The entropy weight of each index is calculated by the Entropy weight method according to its own degree of variation, and then it is used to modify the weight of each index.

$$
w_{j}=\frac{1-S j}{\sum_{j=1}^{n}(1-S j)}
$$

\subsection{Construction of dynamic comprehensive evaluation model based on PSR and DCE}

PSR (Pressure-State-Response) conceptual model was originally proposed to analyze the relationship between environmental stress, current situation and response. DCE (Dynamic Comprehensive Evaluation) is used to analyze the changing speed and trend of events. The shortage of water resources at a certain time points can be calculated by the index system founded upon PSR model. On the basis of the obtained calculation results, the DCE model is used to calculate changing trend and status of the degree of water resource shortage at different time points[2]. In this paper, we measure the ability of a region to provide clean water to meet its needs by constructing a dynamic comprehensive evaluation model based on PSR and DCE .

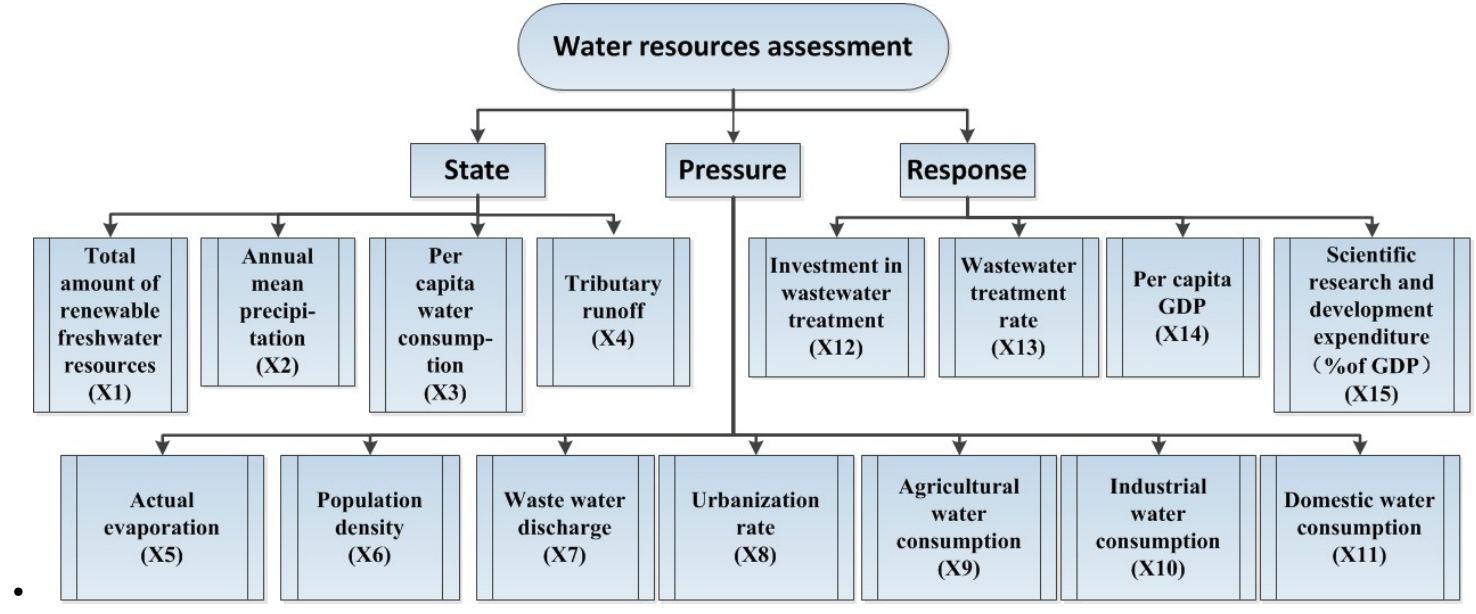

Figure 1: Comprehensive evaluation indexes of water shortage

Establishing Evaluation Index System Based on PSR

The criterion layer of the index system is composed of a pressure index, a state index and a response index. Based on the practical situation in the research area, and the three principles of determining the evaluation index-purposiveness, scientific soundness and typicality, the index system is divided into a number of target layers. The detailed chosen indexes and layers division are shown in the figure below.

- Construction of Dynamic Comprehensive Evaluation Model Based on DCE

Assuming that there are $n$ objects to be evaluated: $(a 1, a 2, \ldots, a n)$, in the continuous m periods ( $t 1$, $t 2, \ldots, t m)$. $a_{i j}$ refers to the value of the $\mathrm{i}-$ th index at a certain time (using $j$ ) which means it is able to be replaced by real statistic. 
In this paper, we only focus on an abstract model, thus we can use the following time sequence information matrix:

$$
\mathbf{A}=\left[a_{i j}\right]_{n \times(m+1)}=\left(\begin{array}{ccc}
a_{11} & \cdots & a_{1 m+1} \\
\vdots & \ddots & \vdots \\
a_{n 1} & \cdots & a_{n m+1}
\end{array}\right)
$$

Noting the change speed of $a_{i}$ in the period of $\left[t_{j}, t_{j}+1\right]$ as $\mu \mathrm{ij} ;(\mathrm{i}=1,2,, \mathrm{n} ; \mathrm{j}=1,2,, \mathrm{~m})$,a time sequence matrix of spend change can be obtained by calculating the following equation:

$$
\square \square \square \mu_{i j}=\left(a_{i j+1}-a_{i j}\right) /\left(t_{j+1}-t_{j}\right) ;(i=1,2, \ldots, n ; j=1,2, \ldots, m)
$$

thus we can obtain the matrix below :

$$
\mathbf{B}=\left[\mu_{i j}\right]_{n \times m}=\left(\begin{array}{ccc}
\mu_{11} & \cdots & \mu_{1 m} \\
\vdots & \ddots & \vdots \\
\mu_{n 1} & \cdots & \mu_{n m}
\end{array}\right)
$$

If the changing trend of the evaluated object is increase, the corresponding rate of change is positive; if the change trend is decrease, then the value of the corresponding change rate is negative. If the evaluation object is stable, the change rate is 0 .

\section{Establishing Measure Model of Velocity Changing State of Water Shortage Degree}

In a certain period of time, if the rate of change of the object $\mathrm{a}_{\mathrm{i}}$ is stable, and in the information collection mode [2] at several different times, its change in the speed is positive during the time period of $\left[t_{j}, t_{j}+1\right]$, then we can draw the conclusion that the rate of change in the state showed a rising trend . Otherwise, there is a downward trend. Assuming $\mu_{i j}$ and $\mu_{i j+1}$ are the value of changing velocity states at two adjacent time $\left[t_{j}, t_{j}+1\right]$, then the area surrounded by the change of velocity trajectory and horizontal of the measured object $a_{i}$ namely, the global state of $a_{i} i n\left[t_{j}, t_{j+1}\right]$, can be expressed as $m_{\mu}^{i}$ $\left(t_{j}, t_{j}+1\right)$, a dynamic comprehensive average, the following equation is used to calculate it:

$$
m_{\mu}^{i}\left(t_{j}, t_{j+1}\right)=\int_{t_{j+1}}^{t_{j}}\left[\mu_{i j}+\left(t-t_{j}\right) \times\left(\mu_{i j+1}-\mu_{i j}\right) /\left(t_{j}+1-t_{j}\right)\right] d t
$$

\section{Establishing Measure Model of the Trend of Velocity Changing State of Water Shortage} Degree

Let:

$$
\lambda_{i j}=\left\{\begin{array}{cl}
0 & t_{i j}=1 \\
\left(\mu_{i j+1}-\mu_{i j}\right) /\left(t_{j}+1-t_{j}\right) & t_{i j}>1(i=1,2, \ldots, q)
\end{array}\right.
$$

Then we can structure function $a\left(\lambda_{i j}\right)$ on $\lambda_{i j}$, this function is used to indicate the trend of velocity change :

$$
a\left(\lambda_{i j}\right)=\varepsilon /\left(1+e-\lambda_{i j}\right)
$$

$a\left(\lambda_{i j}\right)$ increases with the increase of $\lambda_{i j}$, specially, the value of $a\left(\lambda_{i j}\right)$ will tend to $\varepsilon$ and 0 , when the value of $\lambda_{i j}$ tends to positive infinity and negative infinity respectively. If we assign $\lambda_{i j}$ with 0 , we can achieve $\lambda_{i j}=1$, which represents the balance point. $\varepsilon$ simultaneously, $=2$.

\section{Dynamic Comprehensive Evaluation Model of Water Shortage Degree}

The process of dynamic comprehensive evaluation of is used to consider the combination of the state and the trend of changing velocity. Let $\mathrm{m}_{\mu}^{* \mathrm{i}}\left(t_{j}, t_{j}+1\right)$ as the dynamic comprehensive evaluation value of $\mathrm{a}_{\mathrm{i}}$ in the time period $\left[t_{j}, t_{j}+1\right]$, we can acquire the following equation :

$$
Y_{j \mu}=m_{\mu}^{* i}\left(t j, t_{j+1}\right)=\left(m_{\mu}^{i}\left(t, t_{j}+1\right), a\left(\lambda_{i j}\right)\right)
$$

Applying the principle of Newton's second law $(\mathrm{kma}=\mathrm{F})$ to the dynamic comprehensive evaluation above, we have:

$$
Y_{j \mu}=K_{j} m_{\mu}^{* i}\left(t j, t_{j+1}\right) a\left(\lambda_{i j}\right) ;(j=1,2, \ldots, q)
$$

Where $K_{j}$ is the ratio, $m_{i} \mu\left(t j, t_{j+1}\right), a\left(\lambda_{i j}\right)$ and $Y_{j \mu}$ are similar to $m, a$ and $F$ in equation $F=k m a$ respectively.

Then we let $k_{i}=1$, if the value of is certain, $a\left(\lambda_{i j}\right)$ is proportionate to $Y_{j \mu}$, However, if the value of $Y_{j \mu}$ is certain,$a\left(\lambda_{i j}\right)$ will be inversely proportional to $m_{\mu}^{i}\left(t_{j}, t_{j+1}\right)$. 


$$
Y_{j \mu}=K j m_{\mu}^{* i}\left(t_{j}, t_{j+1}\right)=a\left(\lambda_{i j}\right) \int_{t_{j+1}}^{t_{j}}\left[\mu_{i j}+\left(t-t_{j}\right) \cdot\left(\mu_{i j}+1-\mu_{i j}\right) /\left(t_{j}+1-t_{j}\right)\right] d t
$$

Consequently, we can achieve the Dynamic comprehensive evaluation value of $\mathrm{a}_{\mathrm{i}}$ in the time period of $\left[t_{1}, t_{q}\right]$ is :

$$
m_{\mu}^{* i}=\sum_{i=1}^{q-1} m_{\mu}^{* i}\left(t_{j}, t_{j}+1\right)
$$

If the changing trend of the evaluated object is increase, its dynamic comprehensive evaluation value is positive. If the changing trend of the evaluated object is decrease, then the corresponding dynamic comprehensive evaluation value is negative; if the object is stable, the change rate is 0 [2].

We will use $m_{\mu}^{* i}$ as the outcome of the evaluation model. According to the general statistical principles, the rank of water shortage risk can be achieved after the Normal testing of all the risk indexes [5].

\section{References}

[1]Xiangjuan Zhang ,xin Li, Xiuxia Li, etc. With the analytic hierarchy process to estab- lish the Beijing-hangzhou grand canal in suzhou high-tech zone section of the water environment bearing capacity index system research [J]. Journal of environmental protection and circular economy, 2011, $31(2): 42-44$.

[2]Wai Wang,Xinyi Xu,Hongrui Wang, etc. Based on PSR and DCE integrated model of water shortage degree and the change trend analysis [J]. JOURNAL OF NATURAL RESOURCES, 2015, 30 (10).

[3]Abdin A E,Gaafar I. Rational water use in Egypt[J]. M. El Moujabber, L. Mandi, G. Trisorio Liuzzi, I. Martin, A. Rabi, R. Rodriguez (eds), 2009: 11-28.

[4]MOHAMED ELSHOPKY, MAJOR., EGYPTM.Sc., Commanders and Staff Officers College, Cairo, 2008 B.Sc., Military Academy, Cairo, 1998 THE IMPACT OF WATER SCARCITY ON EGYPTIAN NATIONAL SECURITY AND ON REGIONAL SECU- RITY IN THE NILE RIVER BASIN,2012-01

[5]Zhang Huaichun, Yu Yongmei. Ecological risk assessment and driving factors of water resources in Shanghai city [J]. people’ s Yangtze River, 2013, 44 (15): 86-89. 